\title{
Efficacy of Stimulation at the Jing-Well Points of Meridians
}

\section{Ying-Jung Tseng ${ }^{1 *}$, Chia-Ying Chao ${ }^{2 *}$, Yu-Chiang Hung ${ }^{1}$, Sheng-Feng Hsu ${ }^{3,4}$, I-Ling Hung ${ }^{1}$ and Wen-Long Hu ${ }^{15,66^{*}}$}

${ }^{1}$ Department of Chinese Medicine, Kaohsiung Chang Gung Memorial Hospital and Chang Gung University College of Medicine, No.123,

Dapi Rd., Niaosong Dist., Kaohsiung City 833, Taiwan

${ }^{2}$ Kaohsiung Municipal Chinese Medical Hospital, No.68, Jhonghua 3rd Rd., Cianjin Dist., Kaohsiung City 801, Taiwan

${ }^{3}$ Graduate Institute of Acupuncture Science, China Medical University, No.91, Hsueh-Shih Road, Taichung City 40402, Taiwan

${ }^{4}$ Department of Chinese Medicine, China Medical University Hospital, No.360, Sec. 2, Neihu Rd., Neihu Dist., Taipei City 114 Taiwan

${ }^{5}$ Kaohsiung Medical University College of Medicine, No.100, Shihcyuan 1st Rd., Sanmin Dist., Kaohsiung City 807, Taiwan

${ }^{6}$ Fooyin University College of Nursing, No.151, Chinhsueh Rd., Ta-liao Dist., Kaohsiung City 831, Taiwan.

\begin{abstract}
Objective: To investigate the efficacy of stimulation at the Jing-Well points of meridians in advanced clinical practice.

Methods: Articles including English or Chinese keywords on the Jing-Well points of meridians published between 2001 and August 2012 were sourced from the Cochrane Library, PubMed, and China National Knowledge Infrastructure databases. On the basis of these reports, we explored the modern applications, mechanisms, and efficacy of the Jing-Well points.

Results: Thirty-five related studies, published mainly in Chinese, were identified. Evidence was found to support the use of Jing-Well point stimulation in the treatment of stroke, persistent vegetative status, severe head injury, vascular dementia, Alzheimer's disease, upper respiratory infection, bronchial asthma, hysterical aphonia, postpartum lactation insufficiency, fetal malpresentation, dysmenorrhea, acne, sudden deafness, sleeping disorders, and post-chemotherapy nausea and vomiting.

Conclusion: Diseases associated with the 12 meridians and meridional dermomeres (十二皮部) can be treated by stimulating the related Jing-Well points. Stimulation of all the Jing-Well points can activate and restore function in the damaged brain. Rigorous high-quality trials are needed to improve the level of evidence on their effectiveness and safety.
\end{abstract}

\section{Introduction}

The Jing-Well points are 12 acupoints located at the tips of the fingers and toes, except for Yongquan (KI1), which is located on the sole. These points together constitute the first set of Five-Shu points, next to the nails, and form the origin of the 12 Yin and Yang meridians. The Qi of these meridians intersect at the ends of the extremities, metaphorically resembling their source [1,2]. Most applications of the Jing-Well points are based on the concepts of NeiJing Lingshu (spiritual pivot), from which the fundamentals of traditional Chinese medicine and acupuncture therapy were established. The Five-Shu points together represent the practical application of the branch-root (Biao Ben) and root-knot (Gen Jie) theories in clinical acupuncture. According to the root-knot theory, the Jing-Well points are the root and origin of the movement of Qi and blood in a concrete meridian, and the acupoints at the terminal parts of the 4 limbs are very important in acupuncture therapy [3].

The ancient classical texts, e.g., Zhenjiu Jiayijing (針炎甲乙經), Zhenjiu Dacheng (針尒大成), Beiji Qianjin Yaofang (備急千金要 方), and Yizong Jinjian (醫宗金鑑), have described the use of JingWell points to treat stroke, seizures, tonsillitis, epistaxis, menorrhagia, and other conditions [4]. Before the era of Huangdi Neijing and the discovery of acupoints, moxibustion was uniquely applied to the meridians for treatment of relevant diseases [5].

Skin impedance measurements at Jing-Well points may be a useful objective outcome for monitoring effectiveness of acupuncture treatment in patients with endometriosis [6]. We reviewed the relevant literature on clinical uses of the Jing-Well points over the last 12 years to investigate the efficacy of Jing-Well point stimulation in advanced clinical practice.

\section{Methods}

We searched the Cochrane Library, PubMed, and China National Infrastructure (CNKI) databases for articles published between
2001 and August 2012 by using the following English keywords: "Jing point," "Well point," "Jing-Well points," "Shaoshang," "LU11," "Shangyang," "LI1," "Zhongchong," "PC9," "Guanchong," “TE1," "Shaochong," "HT9," "Shaoze," "SI1," "Yinbai," "SP1," “Dadun," "LR1," "Lidui," "ST45," “Zuqiaoyin,” “GB44," “Zhiyin," “BL67," "Yougquan," and "KI1." We also searched the CNKI database by using Chinese characters “井穴” “少商,” “商陽,” “中衝, “關衝,” “少衝”, “少澤, “隱 白,”“大敦,”“歷兌”, “管陰”, “至陰”, and “涌泉.” The included articles, with English abstracts, were related to clinical acupuncture studies over the recent 12 years.

\section{Results}

Among the 245 articles (70 in English and 175 in Chinese) obtained in our search, 210 were excluded because their title or abstract indicated that they were not relevant or because the articles described animal studies. The preliminary results sourced mainly from Chinese reports are described, as only few English articles are available. Only 3 articles described randomized, controlled trials, and all 3 articles were in Chinese [7-9]. We found that many kinds of symptoms were managed by stimulating a single point or combination of points, including all the 12 Jing-Well points, which are illustrated in Figure 1. The stimulation methods included acupuncture, moxibustion, bloodletting puncture, laser acupuncture, electroacupuncture, acupressure, and sticking formula.

"Corresponding Author: Dr. Wen-Long Hu, Department of Chinese Medicine, Kaohsiung Chang Gung Memorial Hospital and Chang Gung University College of Medicine, Kaohsiung, Taiwan; E-mail: oolonghu@gmail.com

\#: Contributed equally with corresponding author.

Citation: Tseng YJ, Chao CY, Hung YC, Hsu SF, Hung IL, et al. (2015) Efficacy of Stimulation at the Jing-Well Points of Meridians. Int J Nurs Clin Pract 2: 121. doi: http://dx.doi.org/10.15344/2394-4978/2015/121

Copyright: (c) 2015 Tseng et al. This is an open-access article distributed under the terms of the Creative Commons Attribution License, which permits unrestricted use, distribution, and reproduction in any medium, provided the original author and source are credited. 


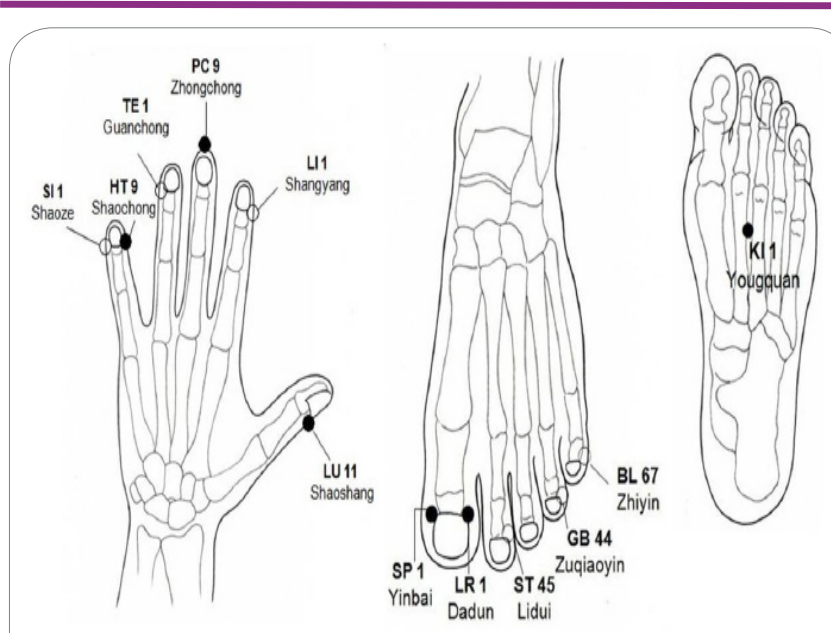

Figure 1: The 12 Jing-Well points; $\circ$ for the Yang meridian and $\bullet$ for the Ying meridian (adapted from $\mathrm{Hu}$ et al., 2011).

\section{Neurological disorders}

\section{Brain damage}

The curative effect of Jing-Well point acupuncture for treating patients with stroke in the convalescent stage is superior to that of ordinary acupuncture [9]. In patients with acute cerebral infarcts, the additional use of Jing-Well points could strengthen the therapeutic effect of conventional acupuncture therapy, especially in improving finger movement and decreasing the level of neurological function impairment [8]. Moreover, acupuncture at the 12 Jing-Well points has a certain therapeutic effect on a persistent vegetative state and shows promising results [10]. Strong stimulation at the 12 Jing-Well points using the half-needling technique has a promising effect in a case of severe traumatic brain injury; with 9 treatments in 3 weeks, the patient's Glasgow Coma Scale (GCS) score improved from E1V1M2 to E4VtM6 [11].

Stimulation of KI1 may help promote recovery of consciousness for patients in long-term comas caused by severe craniocerebral trauma. In a study of patients in comas for more than 3 weeks and GCS scores of 8 or less, electroacupuncture at KI1 and Baihui (GV20), in combination with Western medicine, was effective in improving consciousness [12].

\section{Vascular dementia (VD)}

Acupuncture at the Jing-Well points effectively improves the cognitive function and social behavior of patients with VD, as evaluated by the Mini Mental State Examination (MMSE) and activity of daily living (ADL) scale. Acupuncture in addition to conventional medicine is an effective therapeutic protocol for patients with VD and improves their quality of life [7]. The therapeutic efficacy in cases of VD varies according to the Jing-Well points stimulated; stimulation at HT9 and PC9 yielded better results [13].

The distinct cerebral activation was observed during low-level laser acupuncture (continued wave and $10 \mathrm{~Hz}$-modulated wave stimulation) at KI1 of the left foot in healthy right-handed volunteers [14]. Most of the activated areas, observed by functional magnetic resonance imaging, were involved in the functions of memory, attention, and self-consciousness. The cerebral hemodynamic responses to two laser acupuncture stimulation modes and its mechanism is based on not only afferent sensory information processing but also alteration of hemodynamic properties during external stimulation.
The key factors influencing the effects of acupuncture treatment at the Jing-Well points on VD were explored. The therapeutic intervention (acupuncture) had the most significant influence on the effects against VD. The therapeutic frequency had the least influence. The best therapeutic regimen was as following: acupuncture treatment starting within 1 month of the onset, administered once or twice daily, and 2 therapeutic courses (10 times/course) [15].

\section{Others}

PC9 has even been applied by acupuncture for the treatment of infantile morbid night crying in 100 cases with beneficial results. Pricking the point to cause bleeding can clear heat and purge fire. Thus, it can relieve mental stress and help stop night crying. The therapy is simple and safe and was Jing-Well tolerated by the infants [16]. KI1 and GV20 have been clinically applied in primary trigeminal neuralgia, lower limb pain due to varicose veins, and Meniere's disease [17].

\section{Respiratory disorders}

The primary indication of LU11 stimulation is related to disorders of the respiratory system. This approach can be applied to treat laryngitis, bronchial asthma, and hysterical aphonia [18]. A bloodletting puncture at LU11 is safe and effective for symptomatic treatment of an upper respiratory infection [19]. In children with laryngospasms after tracheal extubation, acupressure on LU11 with the thumb can prevent and relieve the symptoms [20].

In addition to LU11, KI1 also contributes to the treatment of respiratory disorders. Bilateral KI1 stimulation with Biyankang tablet (a Chinese herbal formula) therapy is safe and as effective as $0.5 \%$ Ephedrine naristillae for treating infantile nasal obstruction [21].

\section{Gynecological and obstetric disorders}

\section{Lactation}

SI1 is usually applied for increasing lactation after childbirth. Some studies evaluating the clinical effect of electroacupuncture at SI1 compared with LI1 on hypolactation showed that the mammary filling degree, lactation amount, and prolactin level were better in the SI1 group. Therefore, electroacupuncture at SI1 can not only increase the serum prolactin level but also improve postpartum lactation [2224].

\section{Fetal malpresentation}

Acupuncture, electroacupuncture, laser acupoint stimulation, or moxibustion at BL67 could correct abnormal presentation of the fetus during the last trimester of pregnancy $[25,26]$. Stimulation of BL67 is also helpful for accelerating cervical dilation to achieve pain relief and analgesia during labor; this treatment is effective in about $75 \%$ of the patients $[27,28]$. Bilateral acupuncture plus moxibustion at BL67 was more effective than observation in correcting fetal breech presentation at 33-35 weeks of gestation in Italian women [29]. Therefore, the application of BL67 for correcting fetal malpresentation may be a relatively simple, efficacious, and inexpensive method associated with a lower percentage of operatively completed deliveries [25]. However, another study of a non-Chinese population does not support either the effectiveness or the ineffectiveness of moxibustion in correcting a breech presentation. These results underline the methodological problems in evaluating a traditional treatment in a different cultural context [30]. 
Some evidence suggests that the use of moxibustion at BL67 may reduce the need for oxytocin. When combined with acupuncture, moxibustion may result in fewer births by cesarean section; when combined with postural management techniques, it may reduce the number of noncephalic presentations at birth [31].

\section{Dysmenorrhea}

Acupuncture and moxibustion at BL67 has a promising therapeutic effect on primary dysmenorrhea. Needling at BL67 can transmit the message to the abdomen and the uterus along the course of the kidney meridian so as to restore and regulate Qi and blood circulation in the uterus to relieve pain [32].

\section{Skin diseases}

Bloodletting puncture at ST45 and LU11 has a better effect than that of a Danshentong capsule for treating acne [33].

\section{Other reports}

Stimulation of KI1 may have a therapeutic effect on sudden deafness and sleeping disorders. Acupuncture and moxibustion at KI1 had a superior therapeutic effect on sudden deafness, as compared with conventional medicine [34]. Valerian acupressure at KI1 improved the sleeping time and quality of severely ill patients in the intensive care unit (ICU) who experienced sleeping disorders [35].

In patients with hepatic cancer receiving cisplatin chemotherapy, electroacupuncture at KI1 could prevent and improve the symptoms of nausea and vomiting [36].

The history of nursing is very old. To begin with, nursing is one of the universal occupations of human beings; it is a technique that anybody can acquire by experience and by receiving practical guidance; it is in the nature of humans. Nurses can consider that Nightingale's definition of the concept "knowledge of nursing" points to the breadth of the field of nursing that differs from medicine, and to a discipline which is widely opened to common people [1].

\section{Discussion}

Stimulation at the Jing-Well points of meridians can clear heat, allay excitement, prompt Qi, activate blood, and dredge the meridians. Acupuncture and exsanguinations at these points can clear stagnant Qi and blood within the meridians, adjust organs, promote harmony within organs, un-impede meridians, balance blood and Qi, and promote harmony of Yin and Yang, thus curing disease [1]. According to the theories based on NeiJing Lingshu, the point where energy or Qi arises is a Jing-Well point. Among the Five-Shu points, the JingWell points control the fullness of the subcardiac region and disease in solid organs. These points are widely used for treating many symptoms or diseases related to the associated meridians.

The modern applications of the Jing-Well points in neurology may be realized by the graph of the "somatosensory homunculus." The cortical homunculus is a visual representation of the concept of "the body within the brain"- that one's hand or face exists as much as a series of nerve structures or a "neuron concept" as it does in a physical form. The Jing-Well points are distributed in the hands and feet, which occupy much of the area of the cortical homunculus. Therefore, stimulation of these points has a significant influence on the neurobiological phenomena in the brain, which is why it is frequently used for treating symptoms related to consciousness and neurological problems. Bloodletting puncture at the 6 Jing-Well points of the hand is the classical first-aid measure in traditional Chinese medicine, and this approach has been used clinically for thousands of years. This intervention can improve consciousness in patients with small infarct damage and regulate the circulatory system. The regulation includes increasing systolic blood pressure, accelerating the heart rate, and promoting metabolism and blood supply to the brain. These effects promote neuronal fluid regulation to remove harmful free radicals from the blood. Therefore, in acute cerebral infarction, early intervention by acupuncture at the 12 JingWell points can significantly ameliorate cerebral ischemia [1].

There have been many animal studies pertaining to the Jing-Well points that help further research on the mechanisms. Bloodletting puncture at the Jing-Well points might effectively improve the antistress ability of ischemic brain tissue and protective function of the brain. It can also enhance the expression of heat-shock protein (Hsp) 70 in the initial stage of ischemia and increase the resistance of the brain to the exacerbation of ischemic injury [37]. In rats, early bloodletting puncture at the Jing-Well points can also increase c-fos protein expression and improve the antistress ability of neurons to ameliorate and repair ischemic lesions; prevent slow neuronal apoptosis and development of ischemic lesions; and produce a protective effect in brain tissues [38,39]. These findings suggest that bloodletting puncture at the 12 Jing-Well points may have a beneficial effect in the early stage of stroke. Besides, such therapy has been shown to promote the restoration of motor function and improve learning and memory functions in experimental rats with serious cerebral traumatic injury [40]. PC9 might be useful for treating ischemic cerebrovascular disease: after electroacupuncture was performed at PC9 in rats with reversible cerebral ischemia/reperfusion injury, superoxide dismutase activity increased, malondialdehyde content in brain tissues decreased, and Hsp70 expression was enhanced [41]. Electroacupuncture at LI1, PC9, ST45, and GB44 can reduce the active calmodulin level induced by ischemia/reperfusion injury in rats [42]. Strong electroacupuncture stimulation of KI1 could antagonize the endotoxin thermolysis-induced effect on electrical activities in preoptic and anterior hypothalamus heat-sensitive neurons in rabbits, which may be initiated by the activation of high-threshold thin nerve fibers in the KI1 region [43]. Transcutaneous electrical nerve stimulation (TENS) of KI1 in rats showed that down regulation of extracellular signal-regulated kinase- 2 phosphorylation and c-fos protein expression are involved in the TENS-related inhibition of complete Freund's adjuvant-induced thermal hyperalgesia [44]. The significant increase in the rats' pain threshold by TENS of KI1 suggests that acupuncture may involve the integration of pain processing in multiple brain regions and have inhibitory effects on pain by modulating the release of neurotransmitters and hormones [45].

Electroacupuncture at the Jing-Well points reduced expression of the apoptosis factor Bax in the hippocampal CA1 region in rats, which may be part of the mechanism underlying the improvement in VD [46]. Furthermore, electroacupuncture at PC9 and KI1 significantly improved learning function, enhanced the neurological deficit score, and increased cerebral blood flow in rats with VD, thus helping in the recovery of the conducting cycle related to learning and memory [47]. Besides, this procedure decreased the changes and death of neurons in the hippocampal CA1 area of rats with VD [48]. In rats with VD, electroacupuncture at LI1, PC9, HT9, and KI1 remarkably improved memory impairment and decreased nitric oxide levels in both brain tissue and serum. Inhibition of the overproduction of nitric oxide and its relative neurotoxic effect may be one of the underlying 
Citation: Tseng YJ, Chao CY, Hung YC, Hsu SF, Hung IL, et al. (2015) Efficacy of Stimulation at the Jing-Well Points of Meridians. Int J Nurs Clin Pract 2: 121. doi: http://dx.doi.org/10.15344/2394-4978/2015/121

Page 4 of 5

mechanisms [49]. Electroacupuncture at KI1 and GV20 improved the learning and memory capacity of amyloid precursor protein (APP)-transgenic mice that may be related to its effects in increasing the production of acetylcholine and lowering the levels of APP and amyloid- $\beta$ in the brain [50]. The ultrastructural basis of the improvement in Alzheimer's disease (AD) induced by electroacupuncture at KI1 and GV20 in transgenic mice was investigated. The results suggested that electroacupuncture effectively improved the learning and memory capacity of APP-transgenic mice with $\mathrm{AD}$ and alleviated the pathological changes in neurons of the hippocampal CA1 region, which may be one of the mechanisms underlying the improvement in $\mathrm{AD}$ by electroacupuncture [50]. Electroacupuncture at KI1 and GV20 protected cholinergic neurons in the hippocampus of aged rats with $\mathrm{AD}$ by promoting the synthesis of $\mathrm{c}$-fos protein and increasing the expression level of nerve growth factor (NGF) [51,52].

To explain the underlying mechanisms, biochemically, bloodletting puncture at the 12 Jing-Well points can facilitate the ion pumps of cellular membranes, suppress the calmodulin activity induced by cerebral ischemia, and increase the partial pressure of oxygen in ischemic cerebral tissue to reduce the anoxic state of the brain. With respect to hemodynamics, the procedure could facilitate blood flow in ischemic cerebral tissue and improve intracranial blood flow velocity. Electrophysiologically, the procedure could accelerate heart rate and improve mental activity in patients with stroke. Besides, laser irradiation of the Jing-Well points could improve nail-fold microcirculation [53].

\section{Conclusion}

Diseases associated with the 12 meridians and meridional dermomeres (十二皮部) (Figure 2) can be treated by stimulating the

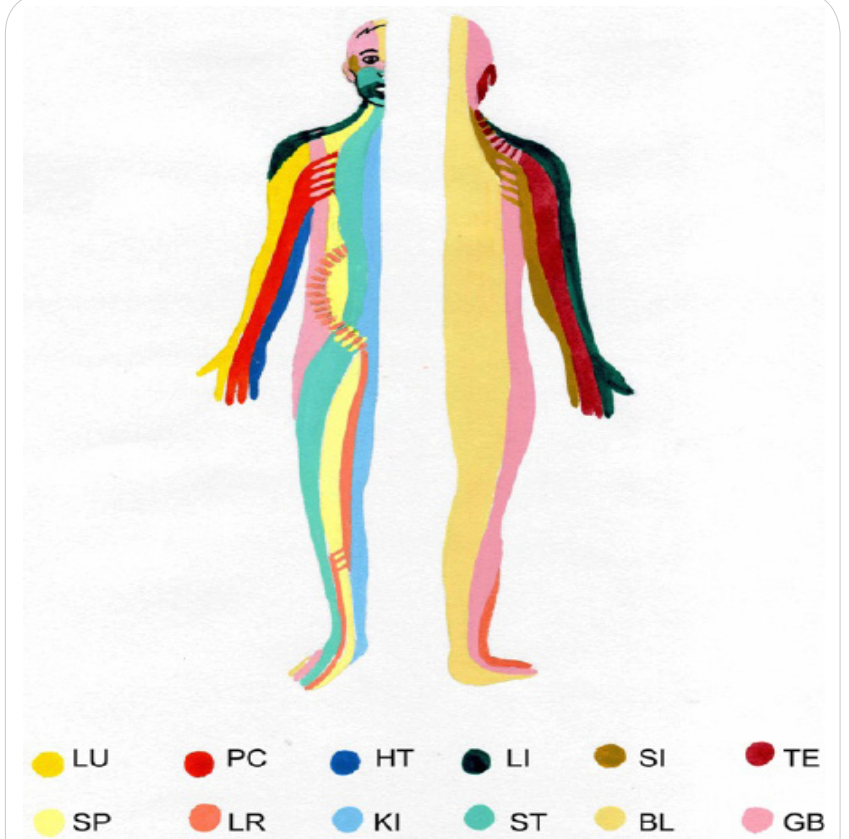

Figure 2: The 12 meridional dermomeres.

relevant Jing-Well points. Stimulation of all the Jing-Well points can activate and restore function in the damaged brain. This method is potentially useful as an alternative or complementary treatment in modern medicine. Rigorous high-quality trials are needed to improve the level of evidence, especially on effectiveness and safety.

\section{Competing Interests}

The authors declare that they have no competing interests.

\section{Author Contributions}

YJT, CYC and WLH contributed in study design, data collection and data analysis. YJT, CYC, WLH, YCH and ILH contributed in manuscript preparation. SFH contributed in manuscript revision.

\section{Acknowledgment}

The authors thank Mr Jui-Hsin Chen for help in manuscript preparation.

\section{References}

1. Hu WL, Hung YC, Chang $\mathrm{CH}$ (2011) Acupuncture for Disorders of Consciousness - A Case Series and Review. In: Saad M (Ed)Acupuncture - Clinical Practice, Particular Techniques and Special Issues, InTech press, Croatia, pp. 5-18.

2. Jin L, Li GL, Guo Y, Jin J (2010) The research progress of clinical application on Jing points in recent 10 years. Journal of Clinical Acupuncture and Moxibustion 7: 66-68.

3. Tang WH (2004) Combined discussion on cycling (Liu Zhu), branch-root (Biao Ben), root-knot (Gen Jie) theories of twelve meridians and circulation (Chu Ru) theory of five-shu points. China Journal of Traditional Chinese Medicine and Pharmacy 4:197-199.

4. Li MX (1996) Explore the law of indications of the Five-Shu points. Journal of Beijing University of Traditional Chinese Medicine 2: 51-53.

5. Lin PH, Li JM, Lin ZG (2008) [The TCM physiological viewpoints in the Pre-Qin dynasty as interpreted from the medical texts from Mawangdui]. Zhonghua Yi Shi Za Zhi 38: 19-23.

6. Ahn AC, Schnyer R, Conboy L, Laufer MR, Wayne PM (2009) Electrodermal measures of Jing-Well points and their clinical relevance in endometriosisrelated chronic pelvic pain. J Altern Complement Med 15: 1293-1305.

7. Shi HR, He F, Dou W, Huang BL, Li XM, et al. (2009) Clinical effect of acupuncture therapy with Jing point for vascular dementia. Journal of Fourth Military Medical University 7: 667-669.

8. Liu DS, Guo Y, Fu W, Li Y (2008) Study of reinforcement the effect of Piercing Jing-points acupuncture on hemiplegia in acute cerebral infarction. Chinese Archives of Traditional Chinese Medicine 2:430-433.

9. Yu HW, Han W, Chen Y (2006) The influence of Jing-well point acupuncture on SIAS and ADL scores in patients with stroke of convalescent stage. Shanghai Journal of Acupuncture and Moxibustion 9:9-10.

10. Chu HR, Yang J, Zeng YL, Li PF (2003) Observation on the awakening effect of acupuncture the Jing (well) points combined with moxibustion the governer vessel on 6 cases of persistent vegetative status. Journal of Clinical Acupuncture and Moxibustion 8: 64-65.

11. Tseng YJ, Hung YC, Hu WL (2013) Acupuncture helps regain postoperative consciousness in patients with traumatic brain injury: a case study. J Altern Complement Med 19: 474-477.

12. Liu JP, Yang ZL, Wang MS, Shi R, Zhu BP (2010) [Observation on therapeutic effect of electroacupuncture therapy for promoting consciousness of patients with coma]. Zhongguo Zhen Jiu 30: 206-208.

13. Han W, Guo T, Zhang L, Yang J (2012) Specific Study on Puncturing Jingwell Points for Vascular Dementia. Shanghai Journal of Acupuncture and Moxibustion 2: 123-127

14. Hsieh CW, Wu JH, Hsieh CH, Wang QF, Chen JH (2011) Different brain network activations induced by modulation and nonmodulation laser acupuncture. Evid Based Complement Alternat Med 2011.

15. Han W, Zhang L, Guo T, Yang J (2012) Key factors research about influence on the effect of VD by acupuncture treatment of well points. Anhui Medical and Pharmaceutical Journal 8: 1099-1102. 
Citation: Tseng YJ, Chao CY, Hung YC, Hsu SF, Hung IL, et al. (2015) Efficacy of Stimulation at the Jing-Well Points of Meridians. Int J Nurs Clin Pract 2: 121. doi: http://dx.doi.org/10.15344/2394-4978/2015/121

Page 5 of 5

16. Zhao J (2002) Treatment of infantile morbid night crying by acupuncture at Zhongchong point in 100 cases. J Tradit Chin Med 22: 11

17. Lun ZJ, Deng LJ, Ye JY, Wang J, Huang YP, et al. (2009) Experiences of the clinical application of Baihui point in combination with Yongquan point. Journal of Liaoning College of Traditional Chinese Medicine 4: 168-169.

18. Xiao YC (2003) Natures and clinical application of acupoint Shaoshang (LU11). Shanghai Journal of Traditional Chinese Medicine 5:46-47.

19. Liu, J, Ma ZH, Zhang M, Chen LP, Tang LC (2010) Therapeutic effect of bloodletting in ear apex and Shaoshang acupoint in 160 patients with acute pharyngeal tumefaction. Journal of Liaoning College of Traditional Chinese Medicine 12: 154-156.

20. Tang YH, Wu XP, Chen HM (2004) Preventing and treating effect of pressing Shaoshang point with thumb on laryngospasm children after trachea extubation. Modern Journal of Integrated Traditional Chinese and Western Medicine 14: 1845-1846.

21. Xing XM (2008) [Observation on therapeutic effect of Yongquan (KI 1) acupoint sticking therapy on infantile nasal obstruction]. Zhongguo Zhen Jiu 28: 808-810.

22. Wang HC, An JM, Han Y, Huang LN, Zhao JW, et al. (2007) [Multicentral randomized controlled studies on acupuncture at Shaoze (SI 1) for treatment of postpartum hypolactation]. Zhongguo Zhen Jiu 27: 85-88.

23. Wei L, Wang H, Han Y, Li C (2008) Clinical observation on the effects of electroacupuncture at Shaoze (SI 1) in 46 cases of postpartum insufficien lactation. J Tradit Chin Med 28: 168-172.

24. HanY, Wang HC, Zhai GR, Zhang HW, Wei LX, et al. (2006) Observation on the therapeutic effect of electroacupuncture of Shaoze (SI 1) in the treatment of parturients with hypolactation. Zhen Ci Yan Jiu 3: 173-175.

25. Habek D, Cerkez Habek J, Jagust M (2003) Acupuncture conversion of fetal breech presentation. Fetal Diagn Ther 18: 418-421.

26. Li X, Hu J, Wang X, Zhang H, Liu J (2009) Moxibustion and other acupuncture point stimulation methods to treat breech presentation: a systematic review of clinical trials. Chin Med 4: 4 .

27. Allais G, Ciochetto D, Airola G, Schiapparelli P, Bosio A, et al. (2003) [Acupuncture in labor management]. Minerva Ginecol 55: 503-510.

28. Duke K, Don M (2005) Acupuncture use for pre-birth treatment. A literature review and audit-based research. Complement Ther Clin Pract 11: 121 126.

29. Neri I, Airola G, Contu G, Allais G, Facchinetti F, et al. (2004) Acupuncture plus moxibustion to resolve breech presentation: a randomized controlled study. J Matern Fetal Neonatal Med 15: 247-252.

30. Cardini F1, Lombardo P, Regalia AL, Regaldo G, Zanini A, et al. (2005) A randomised controlled trial of moxibustion for breech presentation. BJOG 112: 743-747.

31. Coyle ME, Smith CA, Peat B (2012) Cephalic version by moxibustion for breech presentation. Cochrane Database Syst Rev 5: CD003928.

32. Zhou SL, Lin HF (2008) Observation of therapeutic effects of Zhiyin (BL 67 ) on 110 cases of primary dysmenorrheal with acumoxibustion. World Journal of Acupuncture-Moxibustion 4:17-22.

33. Li XP (2005) Efficacy observation on the treatment of acne by quick puncture bleeding in Shaoshang and Lidui points. Hebei Journal of Traditional Chinese Medicine 6: 429-430.

34. Fan XH, Ding YN, Chang XH, Ouyang YL, Xie Q (2010) [Comparative observation on acupuncture-moxibustion and western medication for treatment of sudden deafness]. Zhongguo Zhen Jiu 30: 630-632.

35. Chen JH, Chao YH, Lu SF, Shiung TF, Chao YF (2012) The effectiveness of valerian acupressure on the sleep of ICU patients: a randomized clinical trial. Int J Nurs Stud 49: 913-920.

36. Fu J, Meng ZQ, Chen Z, Peng HT, Liu LM (2006) [Clinical observation on electric stimulation of Yongquan (KI 1) for prevention of nausea and vomiting induced by Cisplatin]. Zhongguo Zhen Jiu 26: 250-252.

37. Wang XY, Li JS, Liu GW, Guo Y, Zhang GZ, et al. (2005) Effect of collateral puncture-bloodletting at Jing point on expression of HSP70 protein of cerebral cortex in rats with middle cerebral artery occlusion. Tianjin Journal of Traditional Chinese Medicine 6: 477-479.

38. Wang XY, Li JS, Guo Y, Liu GW, Pan RJ, et al. (2004) Twelve Jing-Well Points on Corticocerebral C-fos Protein Expression in Rat MCAO Model, Shanghai J Acu-mox 12: 39-41.
39. Wang XY, Li JS, Liu GW, Guo Y, Pan RJ, et al. (2004) nfluence of well acupoints blood-letting therapy on apoptosis associated protein of ischemic brain tissue in rats with acute cerebral ischemia. Tianjin Journal of Traditional Chinese Medicine 2:123-125.

40. Zhang QY, Zhou HJ, Meng JG, Li JS, Wang XY (2009) Effect of puncturing Jing-well points on motor function and learning-memory function in experimental rats with critical cerebral traumatic injury. Journal of Traditional Chinease Medicine 7:620-622.

41. Yan XY (2007) [Effect of electroacupuncture on free radical content and HSP70 expression in the brain tissue in rats with cerebral ischemiareperfusion injury]. Zhen Ci Yan Jiu 32: 102-104.

42. Ma HF, Sun H, Ren XJ, Yan J, Ma WZ, et al. (2002) Influence of electroacupuncture at Shuigou and Jing acupoints respectively on Calmodulin activity in rat ischemic cerebral region. Zhen Ci Yan Jiu 2:102104

43. Dong QS, Dong XM, Zhang XQ (2008) [Effects of strong and weak electroacupuncture on endotoxin-induced changes of electrical activities of heat-sensitive neurons in preoptic area and anterior hypothalamus in rabbits]. Zhen Ci Yan Jiu 33: 124-130.

44. Yang L1, Yang L, Gao X (2010) Transcutaneous electrical nerve stimulation on Yongquan acupoint reduces CFA-induced thermal hyperalgesia of rats via down-regulation of ERK2 phosphorylation and c-Fos expression. Anat Rec (Hoboken) 293: 1207-1213.

45. Yang L, Gao XL, Liu X, Yang LX, Li H, et al. (2005) Effects of TENS of Yongquan on functional magnetic resonance imaging and on pain threshold in rats. Radiologic Practice 12: 1087-1089.

46. Liu G, Yang J (2010) Effects of electroacupuncturing at Jing points on apoptosis gene Bax expression in hippocampus CA1 of rats with vascular dementia. Chinese Journal of Traditional Medical Science and Technology 6: 477-478

47. Liu G., Yang J, Xu GS, Zhang CP (2004) Effects of electroacupuncture at Jing points on learning function, neural behavior and cerebral blood flow of rats with vascular dementia. Chinese Journal of Traditional Medical Science and Technology 5: 257-258.

48. Liu G, Yang J, Xu GS, Zhang CP (2003) The effects of electroacupuncture at Jing-points on ultrastructure in vascular dementia rats. Journal of Clinical Acupuncture and Moxibustion 12: 40-41.

49. Shi HR, He F, Dou W, Mi WN, Fan WL, et al. (2010) Effects of electroacupuncture at related Jing points on the memory impairment and nitric oxide in rats with vascular dementia. Practical Journal of Medicine \& Pharmacy 4: 357-359.

50. Xue WG, Zhang Z, Bai LM, Xu H, Wu HX (2009) [Effect of electroacupuncture on the behavior and the expression of amyloid beta-protein, amyloid precursor protein and ChAT in APP $695 \mathrm{~V} 717$ I transgenic mice]. Zhen Ci Yan Jiu 34: 152-158.

51. Xue WG, Ge GL, Zhang Z, Xu H, Bai LM (2009) [Effect of electroacupuncture on the behavior and hippocampal ultrastructure in APP $695 \mathrm{~V} 717$ transgenic mice]. Zhen Ci Yan Jiu 34: 309-314.

52. Tang Y, Yu SG, Luo S, Han T, Yin HY (2006) [Neuroprotective effect of electroacupuncture therapy on damaged cholinergic neurons in rats with Alzheimer's disease]. Zhong Xi Yi Jie He Xue Bao 4: 374-377.

53. Ma HF, Guo CC, Ma WZ, Sui MH (2003) Progress of study on acupuncture and moxibustion of twelve Jing-points for treatment of diseases. Zhen $\mathrm{Cl}$ Yan Jiu 3: 234-236. 